\title{
LOS TEJIDOS ANDINOS, INDICADORES DE CAMBIO: APUNTES SOBRE SU ROL Y SIGNIFICADO EN UNA COMUNIDAD RURAL*
}

\author{
ANDEAN TEXTILES, INDICATORS OF CHANGE: NOTES ABOUT THEIR \\ ROLE AND MEANING AS OBSERVED IN A RURAL COMMUNITY
}

\author{
Eva Fischer ${ }^{1}$
}

\begin{abstract}
Los textiles andinos son artefactos con cualidades excepcionales. Aparte de ser objetos táctiles y, a la vez, medios de transmisión de informaciones socioculturales sobre el estado social y los valores estéticos, por ejemplo, transmiten datos sobre la economía local e información histórica. Mediante los tejidos se establecen relaciones que entrecruzan estos ámbitos. Hoy en día, las telas elaboradas en el telar tradicional ${ }^{1}$ siguen teniendo un papel importante en las sociedades locales andinas. Están presentes en todo momento crítico del ciclo vital y desempeñan una función conservadora que crea identidad. En la última década se pudieron detectar indicios de un real cambio de rol, y éste se manifiesta en el significado de las telas. Estas alteraciones se originan en la situación sociopolítica actual de la región andina, así como en las condiciones económicas locales, influidas tanto por la globalización como por la mundialización ${ }^{2}$. Investigar estos procesos a lo largo de la línea de quiebre que se va formando entre las tradiciones adaptadas y las nuevas perspectivas, no sólo ofrece la posibilidad de recabar datos sobre la alteración y la formación de identidades, sino que, también, permite encontrar soluciones definidas por los actores locales mismos.
\end{abstract}

Palabras claves: sociedad rural andina, tejidos, contexto sociocultural, identidad, proyectos de artesanía.

\begin{abstract}
Andean textiles are artefacts with extraordinary qualities. Apart from being tactile objects and transmission medium for sociocultural information, for example about social status and esthetical values, they transfer data about local economies and historical incidents. The use of textiles creates relationships that crisscross all these domains. Until the present time cloth produced on traditional weaving instruments takes a predominant role in Andean local societies. It accompanies all moments of crisis during the life cycle and fulfils preserving functions that simultaneously form identity. Recently a shift of the textiles' roles can be detected that also is indicated by their importance. This alteration is linked with both, socio-political incidents currently taking place within the Andean region and the local economic conditions influenced by the world system. The investigation of these processes along their break line holds the possibility of data collection with reference to the alteration and the formation of collective and individual identity. Such a study also will offer solutions defined exclusively by the involved local actors.
\end{abstract}

Key words: Andean peasant societies, textiles, socio-cultural contexts, identity, handicraft projects.

De los tejidos andinos se conoce un amplio registro de técnicas. Entre todas ellas, en este artículo sólo serán tomadas en consideración las que actualmente se manufacturan con técnicas en faz de urdimbre, lo que implica el empleo de fibras de lanas de oveja, de alpaca, así como hilados sintéticos. Los datos de campo referidos en este texto (Fischer 1999, 2007) fueron recabados en la comunidad de Upinhuaya situada en los valles interandinos de la región Kallawaya ${ }^{3}$. El acercamiento a la cuestión central será guiado por las pautas sugeridas por Fox (1979:39, comparar Schneider y Weiner 1989: 1-32; Fischer 1999, 2007) quien propone proceder a partir de una perspectiva holística ${ }^{4}$ ofrecida por la antropología social y cultural. Este marco excluye las contemplaciones efectuadas desde la perspectiva de la Historia del Arte. Se pretende sondear una importante problemática en el amplio tema de los tejidos andinos: la del rol y del significado social y económico de los textiles dentro de sus contextos locales y regionales. Debido a que estos tejidos se hallan vinculados íntimamente con la identidad -tanto individual como colectiva-y para posibilitar una mejor comprensión de lo expuesto, este ensayo también requiere un breve recorrido por los rasgos destacados del contexto andino y los conceptos

\footnotetext{
* Ponencia presentada en la Mesa "Textiles tradicionales americanos. Significados de una actividad tradicional en el contexto de la globalización", organizada por Cecilia Pérez de Micou, Liliana Ulloa y Elayne Zorn, en el $52^{\circ}$ Congreso Internacional de Americanistas, Sevilla, 17 al 21 de julio de 2006. La versión final en español fue revisada por Pablo Espinoza.

1 Instituto de Antropología Social, Universidad de Berna, Berna, Suiza. eva.fischer@anthro.unibe.ch
} 
teóricos sobre identidad recientemente desarrollados. Se partirá de un eje netamente socioantropológico y se emplearán los métodos desarrollados por esta disciplina -un procedimiento en el que se combinan, según la necesidad del caso, acercamientos inductivos y deductivos (Lukas 1994:94).

Primero, y a modo de una introducción al tema, se explican los trasfondos particulares andinos y los recientes teóricos. Luego se presentan, tomando como ejemplo la producción textil en la comunidad de Upinhuaya, las facetas más importantes tanto del rol como del significado socioeconómico y tradicional de los tejidos de la región Kallawaya, que se define como una provincia de estilo reconocida por Gisbert et al. (1987). Seguidamente se especifican los rasgos relevantes de los problemas actuales y, a modo de conclusión, se aborda el problema "producción textil tradicional local" desarrollada por los mismos actores.

\section{Contexto Regional Andino y la Formación de Identidad}

En 1532 no sólo se invadieron y se anexaron los territorios de las sociedades andinas, que eran percibidos como heredados de sus ancestros, sino que, de esa época en adelante, también se intentó controlar, alterar y destruir las expresiones socioculturales relacionadas con el pasado mediante normas restrictivas como, por ejemplo, las Ordenanzas del Virrey Francisco de Toledo 5 . Reaccionando a este choque provocado por el violento cambio de paradigma hasta entonces válido, muchos de los grupos rurales y marginalizados se transformaron en verdaderos conservadores de los elementos culturales definidos hoy en día como andinos (Fischer 2007:27; Izko 1986:60). En consecuencia, y siguiendo las necesidades para poder sobrevivir social y culturalmente durante estos períodos hasta las décadas recientes, las sociedades andinas, aparte de las rebeliones y levantamientos bien documentados, desarrollaron una actitud de resistencia indirecta y clandestina. Pasaban una larga etapa relacionándose sólo en algunos aspectos con las sociedades criollas y se desarrollaban como sociedades paralelas en la macrorregión (Albó 1999:776, 781-783, 823; Larson 1999:565, 571, 659, 680 $)^{6}$. La actitud de resistencia también fue apoyada por la estabilidad de las condiciones básicas de producción agrícola en los grupos locales andinos y éstas, por su parte, se encontraban en una relación íntima con el sistema de parentesco y la estructura social. Así que ambos sectores fueron reproducidos y conservados en su conjunto (Godelier 1990 [1984]; Saignes 1993:1122). También se nota una correlación directa entre la agricultura y los tejidos, ya que ambos participan en la construcción y en la reproducción tanto de la realidad social como de las partes ideales de la realidad, y en consecuencia de las identidades individuales y colectivas ${ }^{7}$.

Contemplando estos desarrollos que se efectuaban a largo plazo en la macrorregión andina, desde la posición de Hannerz (1996:66) se trata, en parte, de un proceso de criollización que obliga a sus actores a buscar nuevas síntesis, así como a volver sobre las potencialidades culturales escondidas y la creatividad propia (ver también Abercrombie 1998, 1993:139-142). Sin embargo, algunos autores califican las sociedades del Cono Sur como "mestizadas por completo", aproximación que lleva a acentuar la ausencia de genuinas características culturales, las que generalmente se asocian con lo prehispánico (Müller 1996:83, nota 2). Este tipo de afirmaciones no arribará a resultados válidos, pero nos conduce directamente al tema de los procesos formativos de la identidad, de los saberes, de la transferencia y de las transformaciones de las tradiciones locales (Berger y Luckmann 2004; ver también Appadurai 1999, 2005; Geschiere y van Binsbergen 2003).

La construcción de la identidad siempre va relacionada con la conciencia, tanto individual como colectiva, de sus portadores. La conciencia colectiva se desarrolla interactuando con la identidad étnica, la que se puede definir como la percepción de atributos específicos, originarios de adentro y de afuera del espacio social de un grupo, y considerando su dimensión cualitativa. Observada bajo aspectos de diferencia y de igualdad, la identidad es el eje principal de la organización social. Esto se refiere especialmente a la práctica de los individuos de utilizar las dos categorías contradictorias en su función de instrumentos sociopolíticos e ideológicos. En consecuencia, el estatus del actor envuelto en este proceso y considerando su capacidad de vivir la diferencia muestra importantes perspectivas para el análisis.

La identidad siempre sintetiza elementos fluidos y estables y, en consecuencia, está sujeta a un proceso de transformación permanente (Hall 1981, 1999:299). En el contexto espacial de este estudio que apunta a la región Kallawaya, en general, y a la comunidad 
de Upinhuaya, en particular, algunos elementos con referencias definibles claramente como "cultura de memoria" (White y Jorion 1996) indican cualidades de estabilidad (ver también Berger 1998). Los lenguajes de identidad y de filiación grupal pueden caracterizarse por sus ambivalencias inherentes y en términos de inclusión, acumulación y exclusión de atributos definidos (Augé 1999:57). La definición formulada por Marshall Sahlins (2000:144), en la que se refiere a la identidad percibida como ...a dialogue between the received categories and the perceived contexts, between cultural sense and practical reference... también debe ser tomada en consideración. Estas relaciones son indispensables para la construcción de las identidades políticas, locales y regionales, y la creación de diferencias y de estereotipos.

A continuación, esta temática se aborda desde la intersección de dos ámbitos: el proceso de transición -que en algunos de los casos puede ser definido como un estado de criollización ${ }^{9}$ - y las estrategias locales, que se desarrollan como reacciones a las condiciones supralocales. Los procesos transitorios iniciados en la última década en los Andes bolivianos influyen en las sociedades rurales como un todo $y$, paulatinamente, empiezan a manifestarse en el manejo del sector textil. Éste todavía sigue integrando una parte importante de la cultura material y está relacionado indispensablemente con la vida social y con el modo de vivir andino.

Generalmente, se pueden distinguir dos tendencias del desarrollo actual. Por un lado, la necesidad de disponer de ingresos en forma de dinero en la población rural provoca el deseo de comercializar los tejidos de un modo eficaz. De este modo las prendas simultáneamente están sujetas a un proceso de despersonalización y de folklorización que influye en la comunidad local y en sus vínculos con los tejidos. Por otro lado, definidos como una expresión cultural fundamental de los grupos rurales -cuyos contextos socioeconómicos se encuentran en proceso de cambio- se aprovecha su potencialidad inherente para crear identidades. Esto se lleva a cabo, por ejemplo, mediante las escenificaciones realizadas fuera del contexto local y relacionadas íntimamente con el contexto nacional y la cultura pública. Es este tipo de señales que se utilizan para la construcción de la cultura nacional las que absorben las expresiones locales para sus propios fines (Saignes 1986a:173-212).
Un componente importante de los tejidos andinos es su multifuncionalidad que, hasta la actualidad, ha incentivado y consolidado un nexo estrecho entre la economía, la vida social y los actos rituales. Tanto en el ámbito de la manufactura textil como en el de la producción agrícola se notan, entonces, algunos sectores que participan simultáneamente en la formación de la realidad ideal (Godelier 1990 [1984]:131), la que, al mismo tiempo, precisa de la realidad de la vida social. Los textiles no sólo son utilizados como vestimenta, tanto de uso cotidiano como festivo, sino que también sirven de utensilios indispensables para las actividades agrícolas (Figura 1), las que a su vez exhiben relaciones estrechas con el ámbito ceremonial. Las diferentes formas de uso de los rebozos y ponchos, por ejemplo, como contenedores para almacenar productos agrícolas y para el transporte (Figura 2) o como tapete para el suelo cuando se efectúan los actos rituales, y también de los paños para guardar las hojas de coca, como cubrecabeza de las mujeres y como soporte para disponer ofrendas. Esto muestra categorías de empleo flexibles y no rígidamente estrictas. Tanto la habilidad manual como la creatividad de las tejedoras eran, y siguen siendo, vinculadas íntimamente con su autoestima personal y colectiva. La concomitancia de todos estos elementos forma una parte constitutiva en la construcción de la identidad local, regional y de género. Las alteraciones, ya iniciadas en las estructuras económicas y sociopolíticas locales, observadas hoy en día en las sociedades rurales de la región Kallawaya, e igualmente en la comunidad de Upinhuaya, presentan cambios en la manifestación de identidad e intervendrán tanto en el rol como en el significado de los tejidos ${ }^{10}$.

\section{Contextos Socioeconómicos}

Para comprender mejor el rol y el significado que desempeñan los tejidos en las sociedades rurales, y tomando como ejemplo la comunidad de Upinhuaya, se muestran tres contextos que también pueden servir para averiguar las futuras potencialidades de la producción textil, tanto de la local como de la regional. En ésta, cada categoría de materia prima indica una faceta específica del modo de producción local, así como el contexto económico correspondiente y el más extendido. A continuación se presentarán los ejemplos que enfatizan esta perspectiva: 


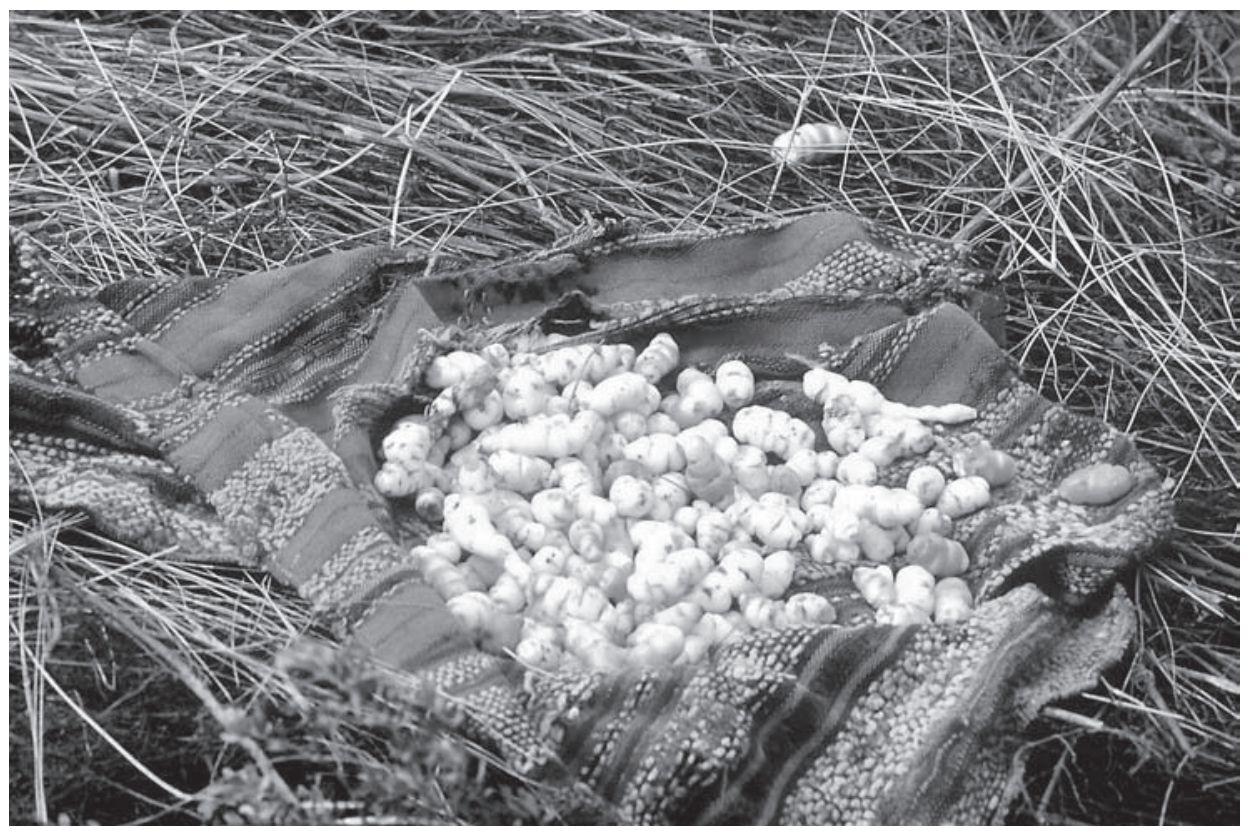

Figura 1. Awayu empleado para secar productos agrícolas, los que para este fin están expuestos al sol. Se trata de un tejido viejo y bastante usado, estado que también se nota por los hilos de zurcir insertados.

Awayu used to dry field crops in the sun. For this purpose old and extremely used textiles, a condition documented by the inserted repair yarns of the displayed textile, are frequently used.

Ejemplo 1: Los hilados manufacturados con las fibras de lana de oveja originaria de la producción local son de baja calidad, pero la lana de los animales criados en las comunidades vecinas Kaata y Kalaya tiene una mejor calidad. Recurriendo a las relaciones de parentesco, tanto las reales como las ficticias, que al mismo tiempo muestran un trasfondo netamente económico, se compensa la escasez de fibras de alta calidad. Dentro del sistema de intercambio local de los productos, bienes y, raras veces, de dinero, las fibras de lana de oveja sólo se pueden intercambiar por azúcar, habas, cebada y hojas de coca. Mientras que las habas y el trigo provienen de la producción local, las hojas de coca y el azúcar remiten a los centros alternativos de la producción agrícola, a los terrenos yungueños ${ }^{11} \mathrm{y}$ a los circuitos migratorios realizados rítmicamente por los comuneros. El azúcar, por ejemplo, es un bien muy apreciado y escaso en las comunidades situadas en la cabecera de valle. Para poder emplearlo como medio de intercambio es necesario vender un producto yungueño -naranjas, café u hojas de coca- y, de este modo, transferirlo a la categoría del dinero. Aunque el dinero es un bien muy estimado en la realidad socioeconómica del grupo local, casi no existe un círculo firme para su

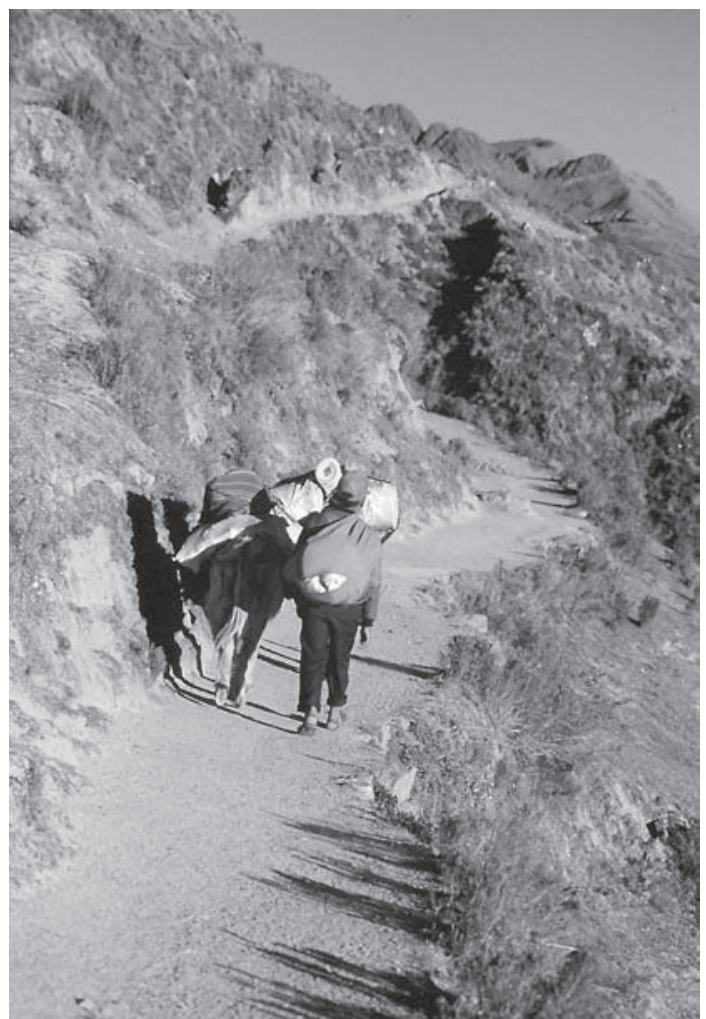

Figura 2. Utilizando el poncho para fines de transporte. Man using his poncho to carry a burden. 
intercambio entre las comunidades regionales ${ }^{12}$. $\mathrm{Al}$ contrario de esto, el intercambio con productos agrícolas permite a las tejedoras efectuar un tipo de transacción directamente bajo su control, ya que las habas y el trigo forman parte del producto excedente ganado mediante la inversión de mano de obra propia ${ }^{13}$.

Ejemplo 2: Las hebras ${ }^{14}$ de urdimbre se manufacturan de distintos materiales, como por ejemplo de las fibras de lana de alpaca y de oveja. Por su cualidad de proporcionar flexibilidad a la tela se emplean las fibras de alpaca, preferentemente de color natural marrón, para la producción de las hebras de trama. Conseguir este tipo de materia prima más los huesos de este animal, llamados wich'uña en aymara, que son indispensables para apretar bien la trama, implica la disposición de relaciones de parentesco, reales o ficticias, con los miembros de las comunidades especializadas en la crianza de camélidos y en la cosecha de sus fibras. Su obtención a través de visitas y viajes con duración de algunos días va conjuntamente con la provisión de carne. Para efectuar estas transacciones se necesitan productos adecuados con valor de intercambio, los que, en este caso, son: copal, habas y cebada.

Ejemplo 3: Las hebras sintéticas sólo se emplean en la formación de urdimbres y se aprecian por sus colores brillantes. Este material no posee una resistencia notable al efecto de los rayos ultravioleta que causan su descomposición y tampoco preserva el calor corporal, tal como logran los materiales de origen animal (Figura 1). Por esta razón se usa con frecuencia para la manufactura de tejidos festivos y para los destinados a la venta en los mercados de artesanía. Las propiedades positivas e indiscutidas de las hebras sintéticas son: la elasticidad y el color intenso, características importantes para el proceso de la producción textil, ya que liberan a

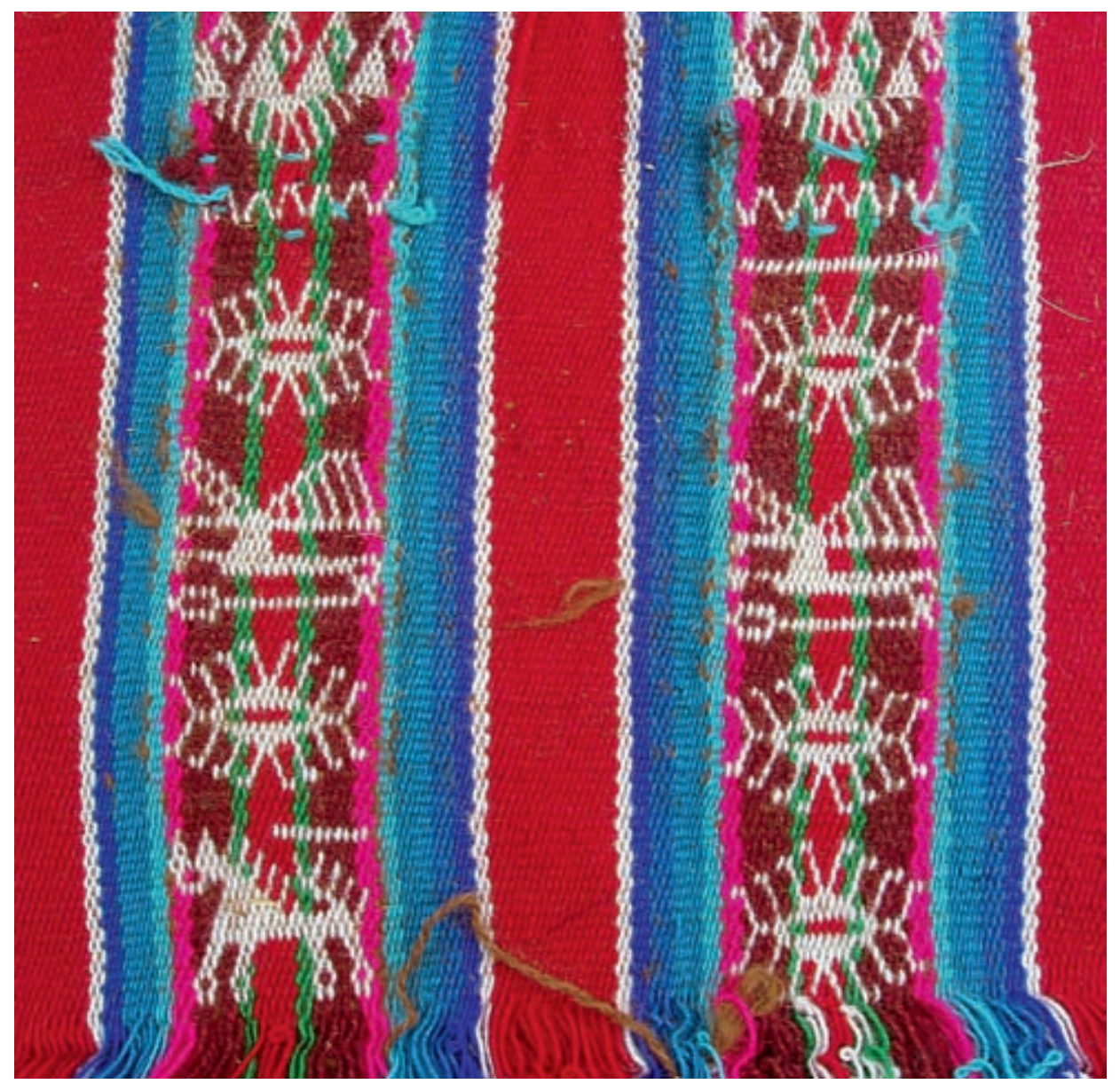

Figura 3. Tejido con la urdimbre constituida por hebras sintéticas.

Textile with synthetic warp yarns. 
las tejedoras de las subsiguientes etapas largas del hilado, torcido, teñido y sobretorcido (Figura 3). A las tejedoras con poca experiencia estas hebras de fibras sintéticas les permiten trabajar rápidamente y también toleran el posible déficit de habilidades manuales. Todos estos factores descritos en los párrafos precedentes influyen tanto en el proceso de la producción textil como en los vínculos de éste con la construcción de la identidad.

Las hebras sintéticas se consiguen mediante la compra, sea por los esposos o por las comuneras mismas, en los mercados locales y regionales. Estos datos muestran claramente la relación estrecha entre la producción industrial de este tipo de materia prima y el dinero como medio único para adquirirla. También remiten al vínculo íntimo entre la producción agrícola local y el círculo de intercambio regional para el aprovisionamiento de las fibras de lanas de oveja y de alpaca. Indican, finalmente, el encaje de dos sistemas económicos: el mundial, presentado por los mercados nacionales, y el regional, fundado en la producción agrícola local. Los ganadores de la intersección de estos dos sistemas mercantiles son los ex terratenientes. Entre los comuneros todavía prevalece el intercambio entre parientes, sobre la venta o compra en los mercados. Esto puede ser tomado como indicio de que, en la manufactura textil, persisten las relaciones recíprocas en cuanto a la organización de la mano de obra, al abastecimiento con materia prima y al préstamo de los instrumentos y utensilios para tejer. También corresponde al concepto del "modo de producción andino".

Los tejidos producidos en la comunidad de Upinhuaya, así como los labrados en las demás comunidades de la región Kallawaya, no sólo siguen siendo objetos relacionados íntimamente con la vida cotidiana, sino también con las esferas sociopolítica y ritual (Figura 4). Dentro del contexto individual, únicamente la muerte del dueño o incidencias negativas pueden interrumpir este proceso de utilización. Dispensar los tejidos completamente de su función significa destruirlos sin dejar material restante y luego tejer un reemplazo adecuado. En la actualidad, en muchos casos, se soluciona el problema mediante la venta, o sea, la transferencia de su valor a dinero y sustituyendo el tejido tradicional por una prenda de proveniencia industrial. Desconectadas de su rol y su significado anterior, estas telas entran como productos al mercado nacional de artesanía y al mercado internacional de arte. Contemplando estos

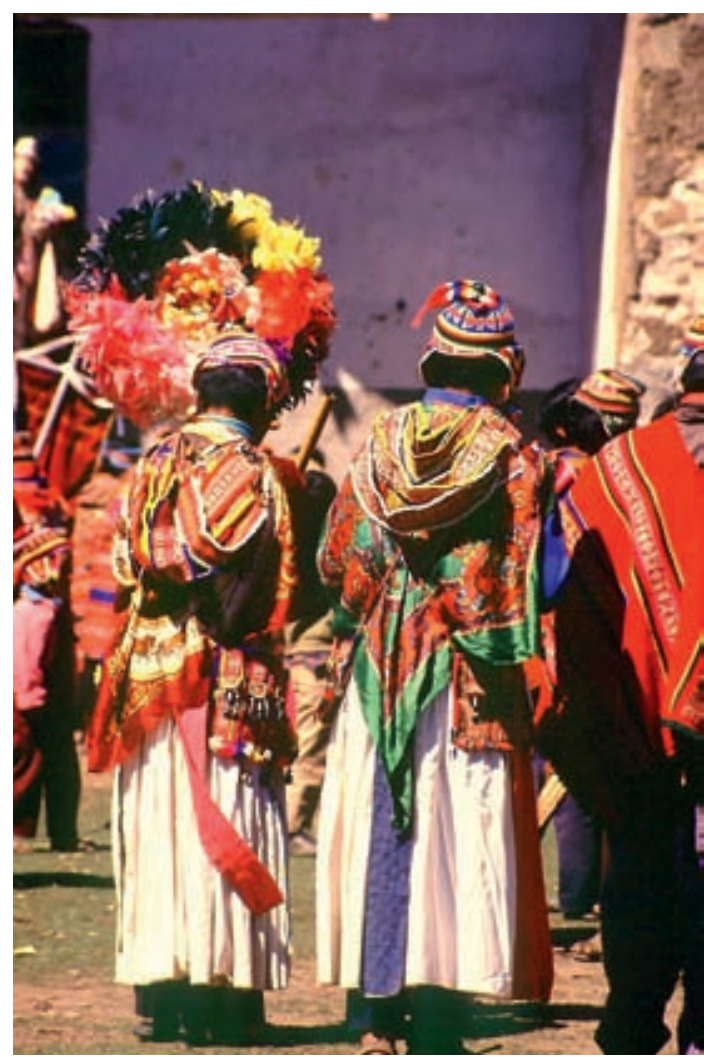

Figura 4. Bailarines con winch'as femeninas puestas sobre su vestuario de fiesta (Fiesta de Sta. Rosa en Kaata, comunidad vecina de Upinhuaya).

Dancers with winch'as, the feminine headbands, put over their feast costumes (The feast of Sta. Rosa celebrated in Kaata, a neighbour community of Upinhuaya).

tejidos, tanto los antiguos coloniales y prehispánicos como los contemporáneos que circulan en este mercado, se nota su cambio de valor: el artefacto relacionado indispensablemente con los diferentes sectores socioculturales de un grupo local (Figura 5) se ha transformado en un bien comercializable cuyo valor de venta depende del mercado regulado por mecanismos externos y mundiales.

\section{Contextos Socioculturales}

Los nexos entre los tejidos y las interacciones sociales se mantienen muy estrechos y se encuentran en un proceso de trascendencia permanente. Los textiles ofrecen un amplio espacio para la creatividad femenina y, con esto, un terreno abierto para expresar las individualidades personales y colectivas. Conjuntamente con la labor textil, acompañan y estructuran las fases vitales femeninas, toman 


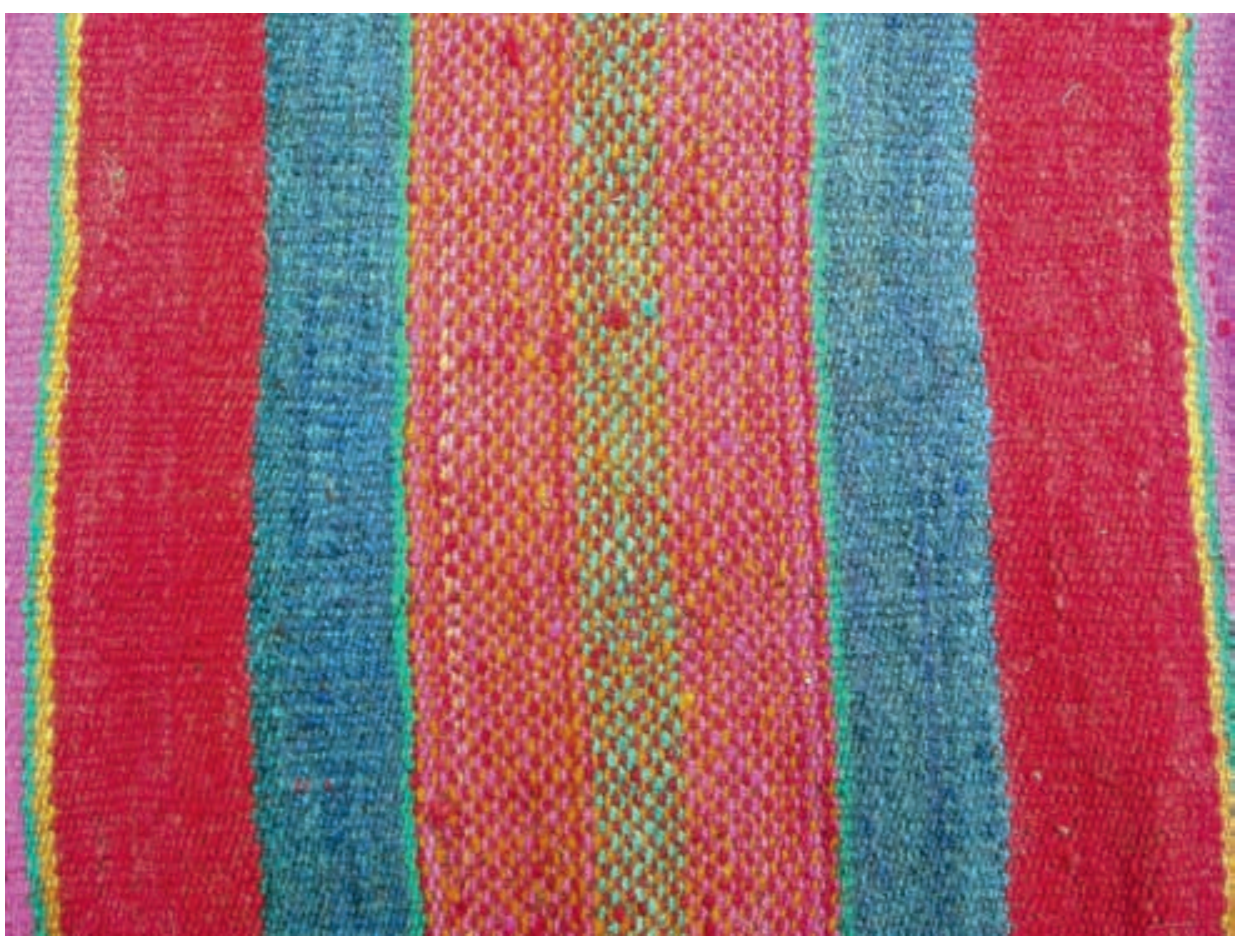

Figura 5. Vista en detalle de un $u n k u$, una camisa ceremonial de varón.

Detail view of an unku, the men's ceremonial shirt.

la función de indicadores del estatus social, de la identidad de género, de las identidades local y regional. En los párrafos siguientes se presentarán tres ejemplos para enfatizar esta perspectiva ${ }^{15}$.

Ejemplo 1: Tanto el proceso de la producción textil en sí como sus productos, los tejidos reflejan aspectos de género y son regidos por las definiciones y atribuciones correspondientes. Todas las labores que forman la cadena del proceso operativo se definen como femeninas. Sin embargo, de niños, muchos varones han sido entrenados por sus madres en el hilado y torcido. Como adultos sólo ejecutan estas tareas para manufacturar las sogas que se utilizan para fijar la carga llevada por los animales. Cuando se trata de un trabajo por encargo, respaldan a sus esposas y, dentro del patio de su casa, participan en el proceso laboral. Debido a la calificación del manejo de los instrumentos y utensilios para tejer como un comportamiento relacionado, exclusivamente, con el ámbito femenino, los varones que hilan y tuercen en público son definidos como homosexuales. Tal evaluación, bajo las circunstancias dadas, también ofrece la oportunidad de traspasar las reglas tradicionales que rigen la división del trabajo por el género y de definirlo mediante la aplicación de criterios personalmente elegidos. Así que, en determinados casos, las personas afectadas empezaron a emplear de un modo muy consciente las categorías opuestas a las asignadas por los padres, las que también han sido adoptadas por la sociedad local.

Correspondiendo con el sistema de pares de elementos con características opuestas, los tejidos tradicionales tienen la función de indicar el género de sus portadores a través de un código estricto en el vestuario (Bourdieu 1974:164): los trajes femeninos y los trajes masculinos. A consecuencia de estas normas, algunas mujeres que decidieron cambiar su género para poder convivir con una compañera utilizaron este lenguaje para sus fines propios y cambiaron su traje femenino por uno masculino. Y algunos hombres, también afectados por estas reglas estrictas, actuaron de forma refleja y se vistieron con el traje femenino. Este modo de proceder para evitar posibles conflictos previsibles dentro del grupo local, con frecuencia lleva a crisis intrafamiliares donde se percibe el género conforme a la definición biológica. Sin embargo, la elección de éste por el mismo individuo afectado lleva a una vida personal socialmente más complacida. Existe, entonces, un espacio de libertad individual dentro de las normas 
rigurosas que definen los roles masculino y femenino, el cual apoya a la cohesión social en el nivel local y permite manifestar la identidad a través del vestuario y de la producción textil.

Ejemplo 2: Si se define traje local como unidad íntegra, el vestuario femenino utilizado en la comunidad visitada forma -tanto mediante sus distintas partes como mediante los signos con carácter narrativo plasmados en los tejidos- un ente icónico. En caso ideal, un conjunto consiste en los tejidos tradicionales winch'a (cinta frontal), urqu (vestido), waka (cinturón) y awayu (rebozo). Hoy la prenda utilizada con menos frecuencia es el urqu porque la manufactura requiere mayor cantidad de tiempo y de materia prima. Las winch'as y las wakas y en tercer lugar los awayus son las prendas que aún persisten en uso, que reflejan la transmisión de los saberes sobre el tejer, que se realiza en línea materna. Contrastando al traje femenino, la vestimenta masculina se compone de pocos tejidos elaborados en el telar tradicional y su elemento más importante es el poncho, que no muestra iconografías tan elaboradas como exhiben las prendas femeninas. Los ponchos son signos de prestigio sociopolítico de los miembros del grupo local.

Los tejidos upinhuayeños, y esto se comprueba por los tejidos producidos en las comunidades vecinas, muestran claramente que existe una definición del grupo como parte integrante de un colectivo más extendido, o sea, como parte de un conjunto compuesto por diferentes grupos locales. En primer lugar, esta determinación se da por los colores y, en segundo lugar, por las distintas formas iconográficas (Fischer 1999, 2007:225) $^{16}$. Tal contexto geopolítico y social, expresado a través de rayas cromáticas, también fue confirmado por los informantes de Cereceda (1978:1028, 2010). A partir de estos datos se formula la hipótesis de que en las sociedades andinas sigue existiendo una legibilidad de las informaciones básicas de un tejido, que van más allá del inmediato contexto local (Femenías 1998; Zorn 1998).

Hoy el significado sociocultural del traje, compuesto por tejidos tradicionales, también se encuentra en vísperas de un cambio. Lo que antes expresaba un alto grado de prestigio en la comunidad y, al mismo tiempo, emitía informaciones sobre pobreza, marginalidad e inferioridad social en los centros político-administrativos, se ha convertido en una expresión de memoria colectiva a nivel local y en una integración folklorizada de los grupos rurales a nivel nacional.
Ejemplo 3: Durante las fiestas locales, en las comunidades se presentan escenificaciones efectuadas por niños vestidos con los trajes tradicionales de acuerdo a su talla. Especialmente los urqus están manufacturados para estos eventos, también se emplean los tejidos de los padres (Figura 6). Este grupo de niños se integra al desfile de los adultos que se visten tanto de prendas de proveniencia industrial como otras producidas en los telares tradicionales. Para los actores, las dos formas de vestirse en estos desfiles -la "tradicional" de los niños y la "moderna" de los adultos- reflejan el nexo con la modernidad dentro del contexto sociocultural local (Figura 7). Se empieza a desarrollar una actitud idealizante del modo de vida tradicional, la que, al mismo tiempo, altera y transforma los significados inherentes a éste, adaptándolos al contexto actual. En consecuencia, los tejidos, que en el pasado también se asociaban con el ámbito ritual y lo sagrado, se transforman en profanos artefactos estéticos que carecen completamente de esta connotación. En tanto, las prácticas rituales correspondientes se encuentran en un proceso de cambio paulatino hacia empresas clandestinas. Son estas referencias las que Taussig (1980:11) toma en consideración cuando menciona "las creencias populares en el contexto de los procesos de transición".

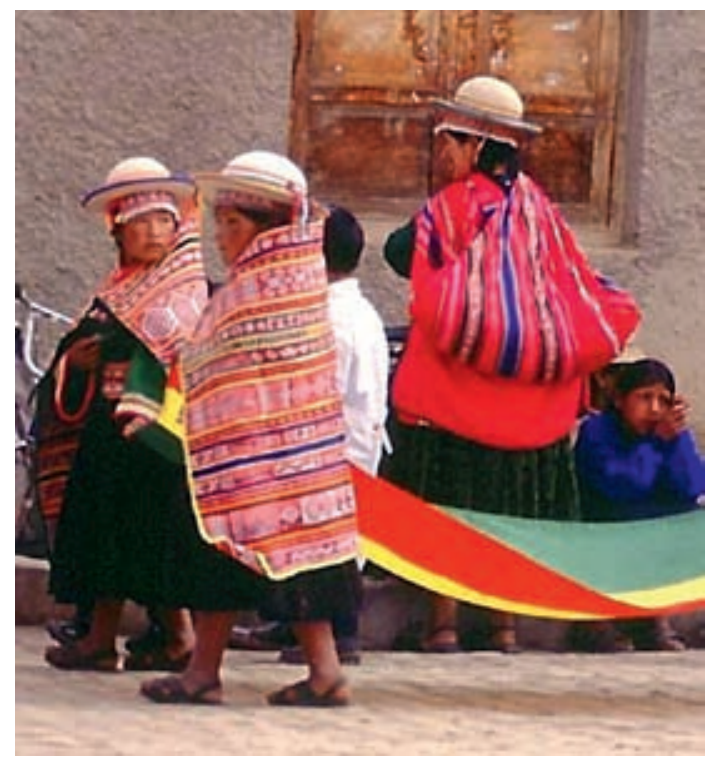

Figura 6. Grupo de niñas vestidas con juegos completos de trajes tradicionales. El urqu está manufacturado de acuerdo a su talla.

Girls wearing traditional clothes. The urqu is manufactured according to their body size. 


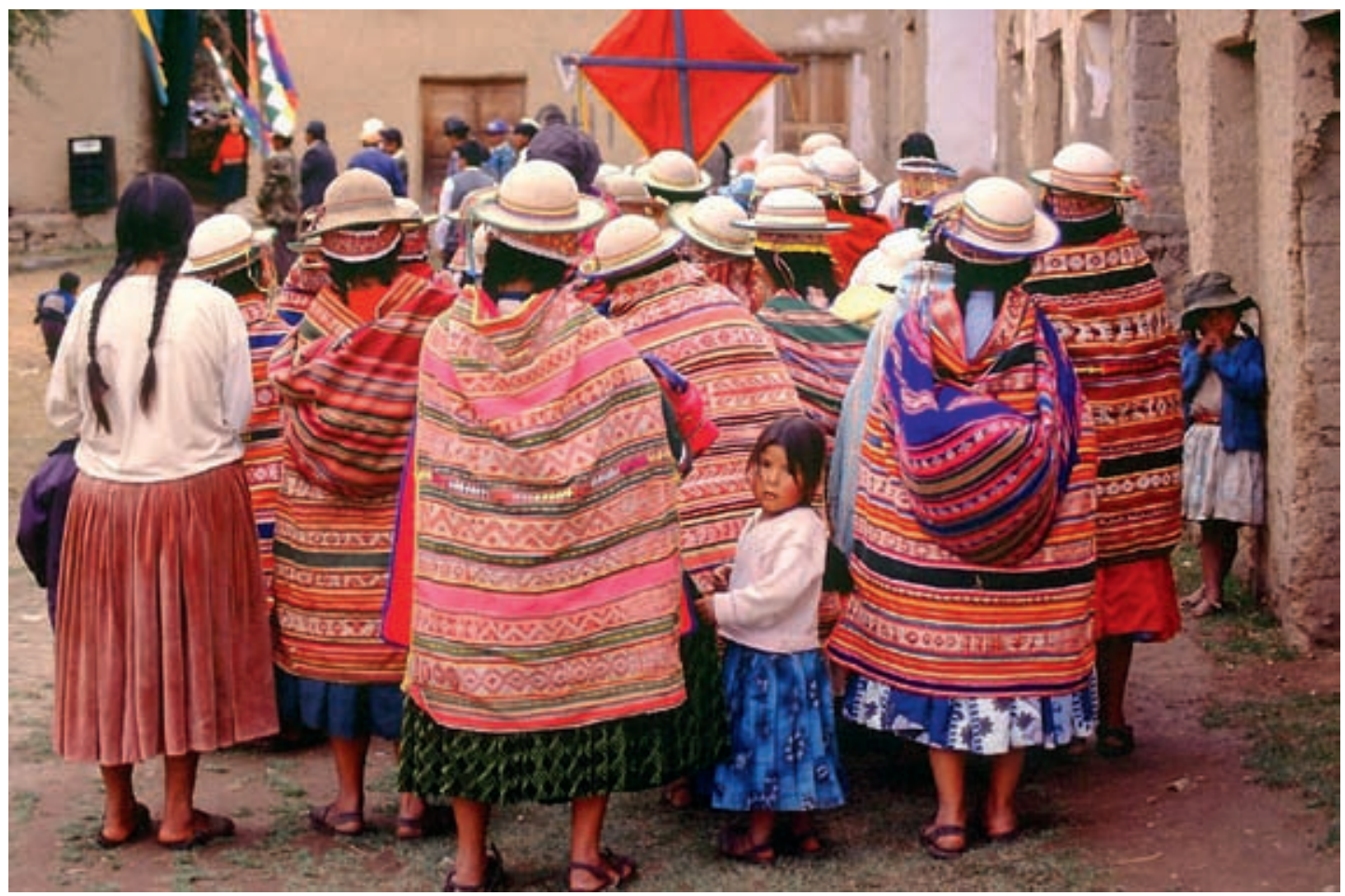

Figura 7. El público, destinatario de las escenificaciones durante los desfiles en las fiestas comunitarias.

The audience, recipient of the performances realized during the feast processions.

\section{Futuro de la Producción Textil Tradicional -Manufactura Artesanal de Objetos sin Función o ¿Producción Integrada al Modo de Vida de la Sociedad Local Moderna?}

Los datos expuestos en los párrafos precedentes muestran claramente que los contextos en los cuales se desarrollaban los tejidos andinos y los que tenían validez para un lapso muy largo, actualmente se encuentran en vísperas de un profundo proceso de transformación. Estas alteraciones intervienen la base económica de los grupos locales e influyen en todos los sectores de la sociedad. En su transcurso, también intervendrán en las labores domésticas y, por esta vía, en el trabajo femenino, en la organización de éste, en la inversión del tiempo para las tareas correspondientes, en la división del trabajo por género y, finalmente, en el tejido y las prácticas relacionadas con él. Los cambios en la manufactura textil también llevarán a la alteración de su significado sociocultural y, en consecuencia, a la cuestión de cómo se realizará su futura incorporación en la vida rural.

Desde los años ochenta y noventa del siglo pasado, en muchas regiones andinas empezaron a florecer iniciativas de producción textil para la venta, tanto en el mercado nacional de artesanía como en el internacional de arte, tejidos tradicionales manufacturados con técnicas de urdimbre. En algunos lugares, en Chincheros (Luis Massa, comunicación personal, 15 de julio de 2003), una comunidad de los alrededores del Cusco, y de aquellas situadas en las islas del lago Titicaca, en Taquile y Amantaní, por ejemplo, los talleres fueron iniciados por los mismos comuneros y resultaron empresas exitosas ${ }^{17}$. Al contrario de estos desarrollos, en la región Kallawaya, especialmente en la comunidad de Upinhuaya, un proyecto de promoción textil sólo funcionó durante el período de tres años en el que permanecieron los promotores e instructores ${ }^{18}$. Considerando el problema con más minuciosidad para poder definir los parámetros, los que posiblemente llevarán a mejores resultados, se debe tratar tanto las preguntas sobre las diferencias entre los distintos proyectos como sus condiciones básicas, las que, finalmente, influyen en la calidad de los frutos del trabajo de las tejedoras locales y del equipo extranjero. Por el momento ignoramos más detalles de los otros casos mencionados y sólo constatamos que en éstos se logró crear y desarrollar 
miniempresas como base económica de los grupos locales. Éstas obedecen tanto al régimen de reglas externas y dictadas por la comercialización del producto y por el mercado regional, como también a las reglas internas y relacionadas íntimamente con la organización sociopolítica de las comunidades. Con el tiempo los dos ámbitos entraron en una síntesis y formaron un nuevo conjunto socioeconómico: la cooperativa del lugar.

En Upinhuaya, según las informaciones obtenidas a través de las entrevistas informales realizadas a las tejedoras, la promoción de la manufactura textil también planteó una esperanza para mejorar la situación económica de las familias, así como la meta de iniciar una producción artesanal a nivel local para el abastecimiento del mercado nacional e internacional. Se planificó responder directamente a la demanda de un sector muy limitado del mercado textil mediante la producción de tejidos teñidos con tintes de origen animal y vegetal. Este cálculo llevó a intentos para revitalizar el conocimiento sobre las sustancias tintóreas tradicionalmente utilizadas para dar color a los kaitus, las hebras empleadas en el tejido. Al mismo tiempo se llegó al primer obstáculo, relacionado con el rudimentario conocimiento tradicional sobre la utilización de estos materiales, el que casi se ha perdido por completo. Además, no se pudo contar con la experiencia de las tejedoras ancianas, porque éstas ya habían aprendido el teñido con colorantes de anilina y no pudieron ofrecer un conocimiento adecuado de las técnicas tradicionales necesarias. El gozo de experimentar hasta obtener resultados deseados y la tolerancia en cuanto a las pérdidas de material y dinero en caso de fracaso llegaron muy rápido a sus límites. Estos vínculos entre la práctica, el ámbito socioeconómico y la creatividad son muy importantes para comprender mejor la actitud tomada por las tejedoras. Las distintas clases de materia prima y sus calidades específicas influyen drásticamente en el éxito del proceso de teñido. Sin embargo, la diversidad de la flora regional y la cantidad impresionante de las plantas tintóreas provenientes, justamente, de la región Kallawaya, constituyen una ventaja competitiva. Recolectarlas, sin duda, requiere una inversión de mano de obra que debe ser bien organizada y realizada siguiendo los parámetros del lugar, que parten de la activación tradicional de mano de obra mediante mink'a y ayni.

$\mathrm{Al}$ contrario de lo inicialmente esperado, el empleo de las sustancias tintóreas de origen vegetal y animal dio como resultado textiles con un registro estrecho en matices carentes de intensidad (Figura 8). Aparte de la diferencia existente entre el régimen cromático definido por las tradiciones

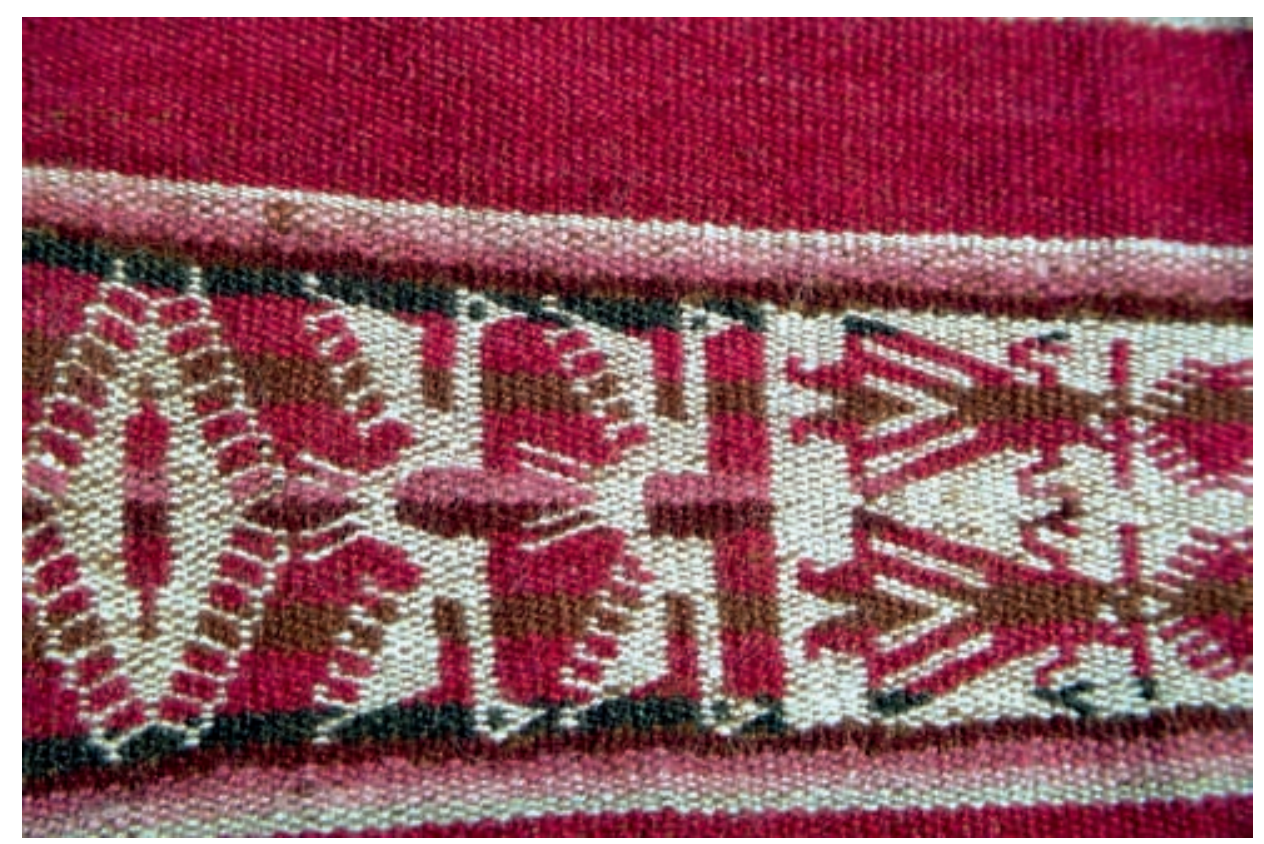

Figura 8. Detalle de un tejido que tiene hebras de urdimbre teñidas con cochinilla, manufacturado en el contexto del proyecto local. Detail of a textile with cochineal dyed warps woven within the local project's context. 


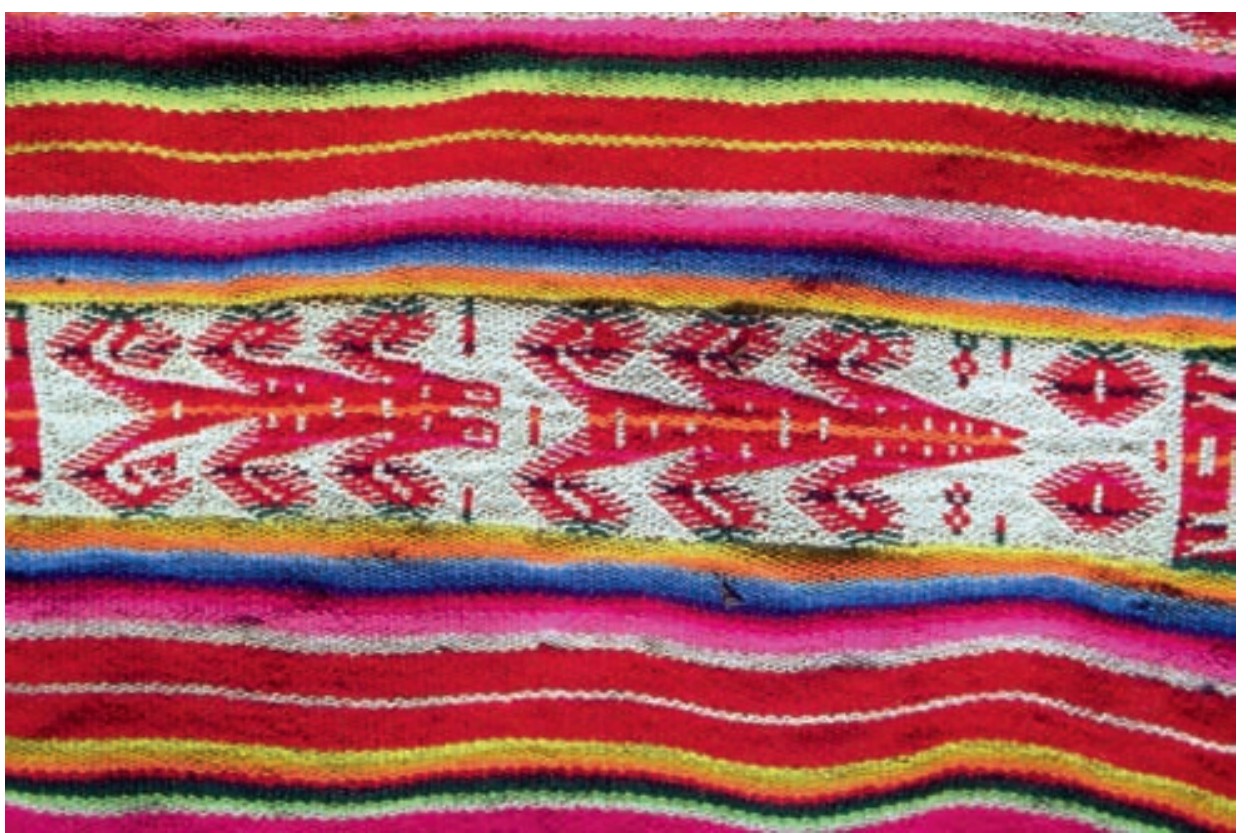

Figura 9. Tejido moderno manufacturado con hebras de urdimbre sintéticas, el que muestra el régimen cromático tradicional de la región Kallawaya y la versión local de Upinhuaya.

Modern textile manufactured with synthetic warp yarn showing the traditional colour scheme of the Kallawaya region and especially the local version of the community Upinhuaya.

locales (Figura 9) y el introducido por las necesidades del proyecto, obviamente no fue posible recurrir al conocimiento experto y tampoco conseguir los fondos para comprar suficientes cantidades de materia prima. Un buen teñido sólo se logra si se agrega suficiente material tintóreo al baño, se dispone del saber adecuado sobre las técnicas de teñido y la experiencia sólida para aplicarlas de un modo eficaz (Figura 10).

Del tinte rojo que se obtiene de la cochinilla ${ }^{19}$, es posible teñir distintos tonos; por ejemplo: rosado intenso, violeta, guindo, rojo brillante, rojo oscuro y rojo anaranjado. El colorante tolera la exposición a los rayos ultravioleta, una cualidad necesaria para alcanzar buenos resultados en el tejido porque el calor del sol aumenta la elasticidad de las hebras de urdimbre durante este proceso laboral.

Para teñir azul se puede utilizar añil, preferentemente el americano ${ }^{20}$. Aunque es un colorante difícil de conseguir y sólo a precios elevados, se pueden compensar estos gastos por el efecto brillante y los matices intensos que suele dar. El proceso de teñido con añil requiere algunos días y su duración depende de la altitud sobre el nivel del mar en la que se está realizando, porque la reacción de la sustancia depositada en las fibras depende de la cantidad de oxígeno del aire ${ }^{21}$. Para todas estas labores se necesita en la fase inicial una/un experta/o tintorera/o que explique los procesos y apoye tanto al aumento del conocimiento como despertar la curiosidad que implica la apropiación del saber (Figura 11).

El proyecto de reunir hilanderas, tintoreras y tejedoras para un trabajo que ellas pudieran ejecutar siguiendo un ímpetu personal y que creara identidad -tanto personal como de grupo local-e ingresos mediante la venta de los textiles provocó en los participantes del proyecto la esperanza de tener un medio a la mano para poder manejar más exitosamente el futuro de sus unidades domésticas. Pero se llegó a resultados insatisfactorios porque la manufactura generó tejidos híbridos que no se podían vender como se había esperado en esta ambiciosa empresa. A causa del formato elegido para los textiles y este modo de crear los diseños, que difiere del estilo tradicional en lo referente al tamaño y a la disposición de los elementos formales, no pueden ser utilizados en la vida cotidiana ni en los actos rituales. Asimismo, no son mercaderías vendibles en el mercado nacional de artesanía, dado el elevado precio que debe cobrarse por el alto costo de los materiales empleados. 


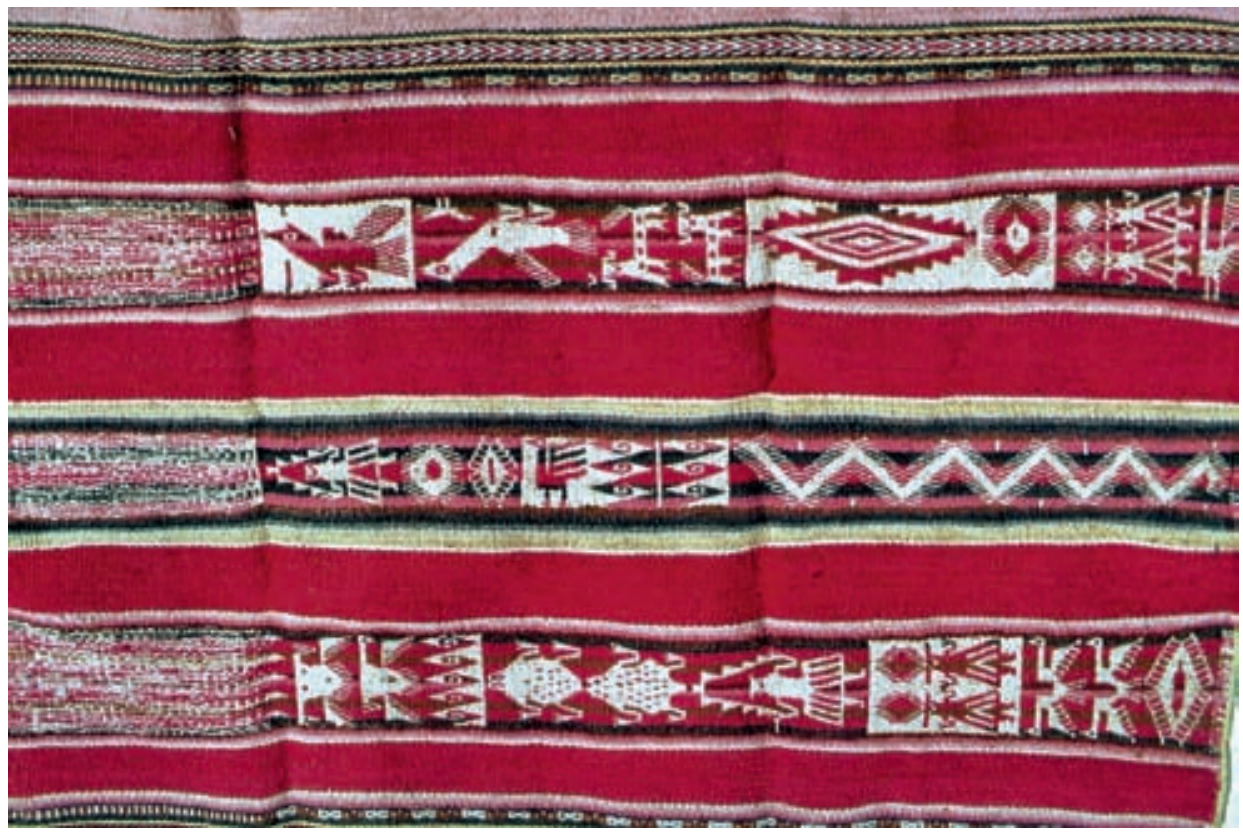

Figura 10. Aspecto general de un tejido manufacturado en el contexto del proyecto local. Se nota su carácter híbrido por la disposición de las rayas y fajas, la que parece al de rebozos, el tamaño rectangular que parece al de los paños de coca, el orden iconográfico de los diseños, y el régimen cromático.

General view of a textile woven within the local project's context. Its hybrid character can be observed clearly by the disposition of the stripes and bands similar to those of the women's shawls, the rectangular shape similar to that of the cloth used for keeping coca leafs, the iconographic order of the designs and colour schemes.

Para analizar los nexos causales de este fracaso -que resultó a costa de las tejedoras y de sus miembros familiares-se debe analizar el problema desde dos perspectivas: la interna, que parte de observaciones de los parámetros locales, y la externa, que toma en consideración las condiciones marco, definidas por los contextos nacionales y mundiales.

Las tejedoras ya han reflexionado sobre su situación y llegado a definir los parámetros para poder manejar su trabajo extradoméstico de un modo más adecuado y exitoso. Entre los factores locales que favorecen el trabajo femenino en el tejido, las tejedoras valoran el fácil acceso a la materia prima para el hilado y el tejido que permite una producción suficiente. Se suman a ello el intenso intercambio de saberes sobre las técnicas, las habilidades dentro de un grupo de simpatizantes y la organización del trabajo que trasciende los límites tradicionales. Sobre eso, trabajar para el propio beneficio también significa desarrollar una rutina, y ésta sólo será posible de obtener cuando la mujeres puedan liberarse de la crianza de los niños y las tareas domésticas, y dedicarse, por lo menos, unas horas al trabajo del tejido. Crear un taller, que cumpla tanto con las condiciones para producir textiles de alta calidad como con las necesidades específicas de las tejedoras, requiere de una buena planificación para la organización, la que también debe orientarse en el ritmo de trabajo integrado con el modo de vida local. Sólo tomando en consideración este trasfondo se pueden solucionar las demás cuestiones como las condiciones en el nivel macro que intervienen en la realidad local y que también lo hacen si las tejedoras logran crear un sector sostenible y autónomo mediante sus proyectos dentro del mercado de artesanía regional y nacional.

Producir un tejido tradicional andino requiere de condiciones específicas, tanto para el proceso creativo como para el de la producción manual. Se trata de una categoría particular de mercadería que no sólo se define por su valor monetario, sino también por su valor cultural, el que se manifiesta en la actitud adoptada por las productoras. Por un lado, ellas quieren seguir practicando el tejido como expresión artística, como la de su memoria colectiva y la de su identidad personal y local, por el otro, requieren de ingresos por su venta. Confrontadas con la contradicción inherente a sus 


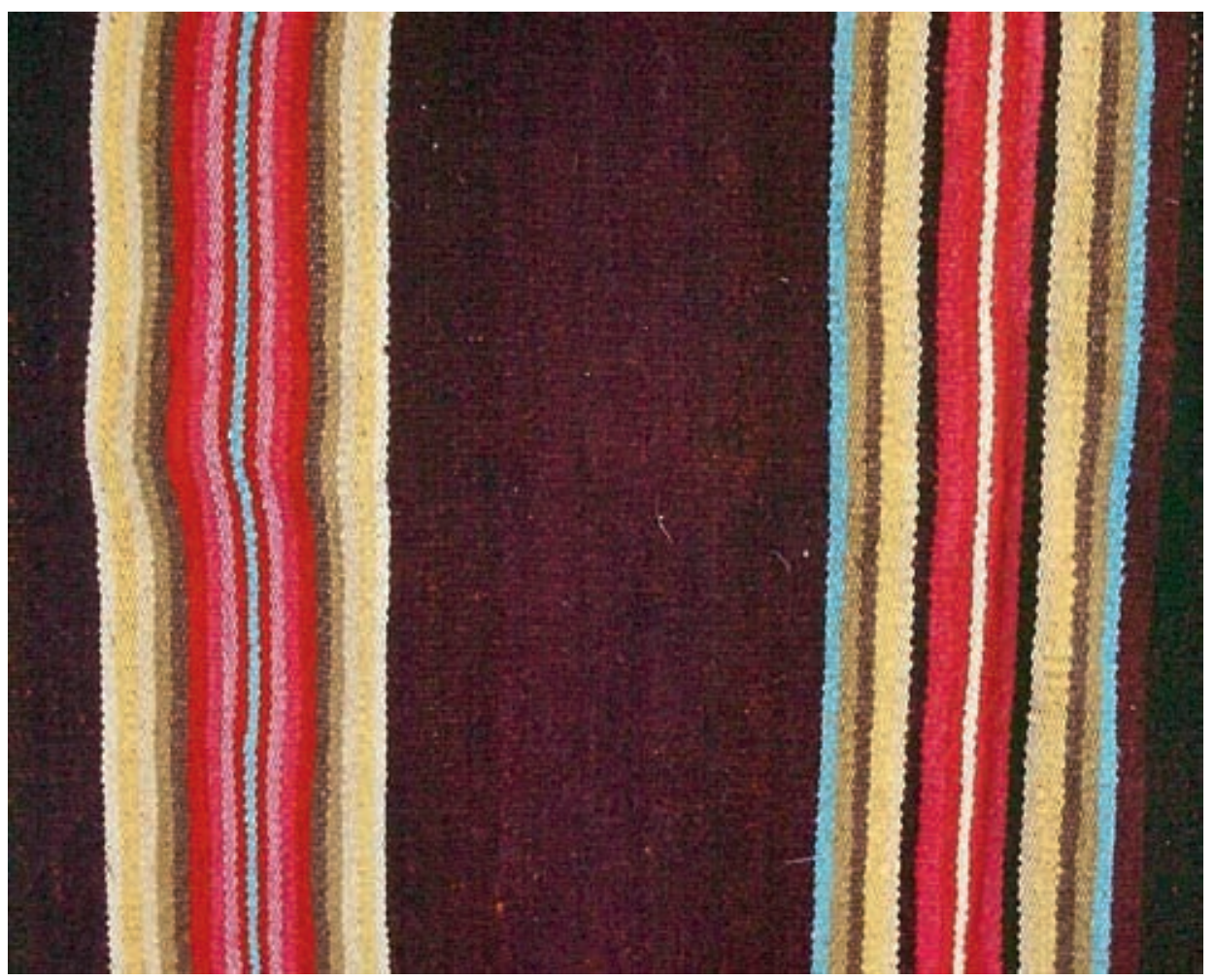

Figura 11. Detalle de un poncho moderno manufacturado en la región Kallawaya que dispone de hebras de urdimbre teñidas con diferentes sustancias de origen vegetal y animal: con cochinilla (rojo brillante, granate y rosado), añil (azul celeste), achiote (amarillo) y t'ola (verde).

Detail view of a modern poncho woven in the Kallawaya region. The textile's warp yarns are dyed with different natural substances of animal or vegetal origin: with cochineal (brilliant red, ruby and pink), indigo (brilliant azure), achiote (yellow) and t'ola (green).

planificaciones, decidieron que, paulatinamente, se deben desarrollar las formas textiles adaptadas a ambas necesidades. En suma, se puede concluir que existen potencialidades en el sistema económico local, las cuales deben explotarse con cuidado por los mismos actores involucrados. Las experiencias recientes conducían a tejer, en la primera fase del proceso reiniciado, pequeños paños con carácter de patrón, los que también podrían servir para apoyar la memoria y la enseñanza en el tejido. Estos artefactos se encontrarían vinculados directamente con la práctica de la manufactura artística desarrollada por las autoras a partir de sus experiencias y del contexto de su modo de vida andino. Estos artefactos son una solución adecuada y constituyen un primer paso hacia la autodefinición y la autonomía económica de las mujeres. Siguiendo fines definidos por las necesidades básicas, ellas manejan los signos sustanciales de la memoria colectiva y dominan las técnicas tradicionales para realizar la producción de la cultura material correspondiente a ésta. Es esa posición que apoya la consolidación de la autoestima de los miembros femeninos del grupo local dentro del contexto de una sociedad moderna del saber lo que, en este momento, se encuentra en una etapa de creación (Figura 12).

Agradecimientos: Quiero expresar mi más sincero agradecimiento a Paulina Brugnoli, Paulina Jélvez, Soledad Hoces de la Guardia y Cecilia Pérez de Micou que me han prestado su apoyo durante la redacción del presente trabajo. Asimismo, mis agradecimientos a los evaluadores anónimos. 


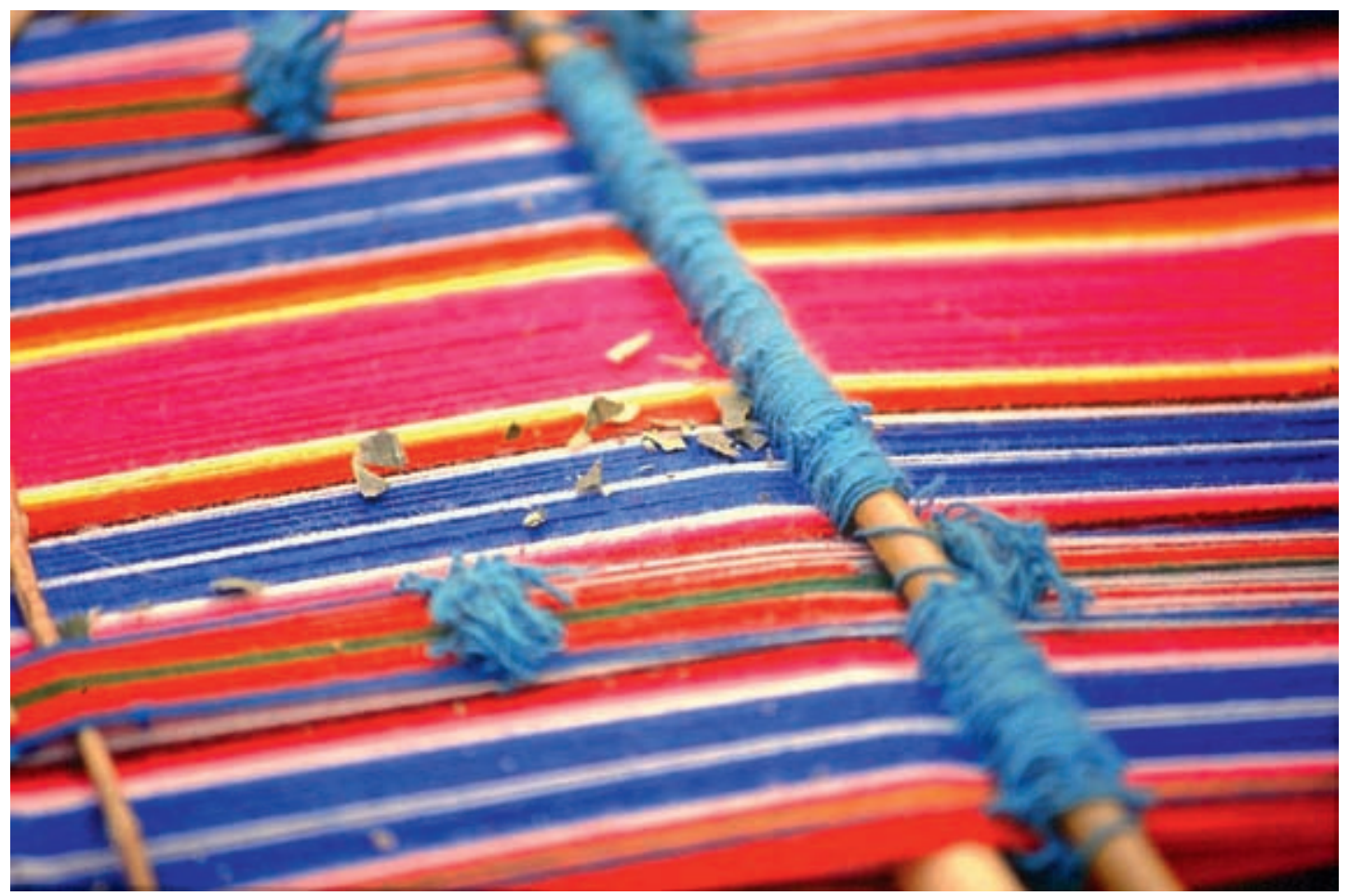

Figura 12. Tejido en proceso de producción con hojas de coca desmenuzadas y esparcidas sobre la urdimbre para activar las fuerzas positivas y obtener un producto de alta calidad.

Unfinished textile with grinded coca leafs scattered on its warp surface. They are supposed to activate positive energies and to favour the creation of a high quality product.

\section{Referencias Citadas}

Abercrombie, $\mathrm{T}$.

1993 Caminos de la memoria en un cosmos colonizado. Poética de la bebida y la conciencia histórica en K'ulta. En Borrachera y Memoria, editado por T. Saignes, pp. 139170. HISBOL, La Paz.

1998 Pathways of Memory and Power. Ethnography and History Among an Andean People. University of Wisconsin, Madison y London.

Albó, X.

1999 Andean People in the Twentieth Century. En The Cambridge History of the Native Peoples of the Americas, editado por F. Salomon y St. Schwartz, pp. 765-871. Cambridge University Press, Cambridge.

Appadurai, A., compilador

1999 The Social Life of Things: Commodities in Cultural Perspective. Cambridge University Press, Cambridge.

Appadurai, A.

2005 Materiality in the future of Anthropology. En Commodification: Things, Agency, and Identities (The Social Life of Things Revisited), editado por W.M.J. van Binsbergen y P.L. Geschiere, pp. 55-62. Lit-Verlag, Münster.

Augé, M.

1999 Mestizo Spaces. An Anthropology for Contemporaneous Worlds. Traducido por A. Jacobs. Stanford University Press, Stanford.
Berger, P.L. y T. Luckmann

2004 Die gesellschaftliche Konstruktion der Wirklichkeit: eine Theorie der Wissenssoziologie. Fischer, Frankfurt am Main.

Berger, P.L.

1998 General observation on normative conflicts and mediation. En The Limits of Social Cohesion. Conflict and Mediation in Pluralist Societies. A Report of the Bertelsmann Foundation to the Club of Rome, editado por P.L. Berger, pp. 352-372. Westview Press, Boulder.

Bertonio, L.

1984 [1612] Vocabulario de la Lengua Aymara. Ediciones Ceres, Cochabamba.

Bourdieu, P.

1974 Zur Soziologie der symbolischen Formen. Traducido por W. Fietkau. Suhrkamp, Frankfurt am Main.

Cajías, M. y B. Fernández

1987 Manual de tintes naturales. Manuales Técnicos 12. Servicios Múltiples de Tecnología Apropiada (SEMTA), La Paz.

Cereceda, V.

1978 Sémiologie des tissus andines: les talegas d'Isluga. Annales 33(5-6):1017-1036.

2010 Semiología de los textiles andinos: las talegas de Isluga. Chungara Revista de Antropología Chilena 42:181-198. 
Feest, C.F.

1999 Technologie und Ergologie in der Völkerkunde. Reimer, Berlin.

Femenías, B

1998 Ethnic artists and the appropriation of fashion: Embroidery and identity in Caylloma, Peru. Chungara 30:197-206

Fischer, E.

1999 Herstellung und Verwendung in musterbildender Kettentechnik gefertigter Textilien der Region Charazani am Beispiel der Dorfgemeinschaft Upinhuaya. Weberei und Gesellschaft andiner Regionen Boliviens. Tesis de doctorado, Universidad de Viena.

2007 Urdiendo el Tejido Social. Sociedad y Producción Textil en los Andes bolivianos. Lit-Verlag, Viena.

Fox, J.J.

1979 Figure Shark and Pattern Crocodile: The foundations of the textile traditions of Roti and Ndao. En Indonesian Textiles. Irene Emery Roundtable on Museum Textiles 1979 Proceedings, editado por M. Gittinger, N. Gwatkin y P. Fiske, pp. 39-55. Textile Museum and Trustees for Harvard University, Dumbarton Oaks, Washington D.C.

Geschiere, P. y W. van Binsbergen, compiladores

2003 The Social Life of Things Revisited. Duke University Press, Duke.

Girault, L.

1969 Textiles Boliviens. Région de Charazani. Serie H-Amérique 4. Musée de l'Homme, Paris.

Gisbert, T., S. Arce y M. Cajías

1987 Arte Textil y Mundo Andino. Gisbert, La Paz.

Godelier, $\mathrm{M}$.

1990 [1984] Lo Ideal y lo Material. Taurus, Madrid.

Hall, S.

1981 Cultural studies: Two paradigms. En Culture, Ideology and Social Process, editado por T. Bennett, G. Martin, C. Mercer y J. Woollacott, pp. 19-37. The Open University Press, Maidenhead.

1999 Cultural identity and Diaspora. En Migration, Diasporas and Transnationalism, editado por S. Vertovec y R. Cohen, pp. 299-314. Elgar Publishing, Cheltenham y Northampton.

Hannerz, U.

1996 Transnational Connections. Culture, People, Places. Routledge, London and New York.

Hardman, M. J., J. Vásquez y J. de D. de Yapita 1988 Aymara. Compendio de Estructura Fonológica y Gramatical. ILCA/Gramma, La Paz.

Izko, J.

1986 Comunidad andina: Persistencia y cambio. Revista Andina 7(1):59-99.

Larson, B.

1999 Andean Highland peasants and the trials of Nation making during the Nineteenth Century. En The Cambridge History of the Native Peoples of the Americas, editado por
F. Salomon y St. Schwartz, pp. 558-703. Cambridge University Press, Cambridge.

Lukas, $\mathrm{H}$.

1994 Tondong-ein ethnologisches Hypermedia-Beispiel. IITF-Schlußbericht für das Forschungsprojekt: Konzept und Implementierung einer sozialanthropologischen Wissensdatenbank mit Bildspeicherung als modellhaftes Fachinformationssystem, Schlussbericht. pp. 93-130. Universidad de Viena, Viena.

Müller, H.P.

1996 Kulturelle Gliederung der Entwicklungsländer. En Weltsystem und Kulturelles Erbe, editado por H.P. Müller, pp. 81-138. Dietrich Reimer Verlag, Berlin.

Sahlins, M.

2000 Culture in Practice. Selected Essays. Zone Books, New York.

Saignes, $\mathrm{T}$.

1985 Quiénes son los kallawaya? Nota sobre un enigma histórico. En Los Andes Orientales: Historia de un Olvido. Estudios Históricos 2. Centro de Estudios de la Realidad Económica y Social, Cochabamba.

1986a Las sociedades de los Andes frente al Estado republicano. En Estados y Naciones en los Andes, editado por J.P. Deler e Y. Saint-Geours, pp. 173-212. IEP, Lima.

1986b En busca del poblamiento étnico de los Andes Bolivianos (siglo XV y XVI). Avances de Investigación 3. Museo Nacional de Etnografía y Folklore, La Paz.

1993 "Estar en otra cabeza": Tomar en los Andes. En Borrachera y Memoria. La Experiencia de lo Sagrado en los Andes, editado por T. Saignes, pp. 11-22. HISBOL, La Paz.

Soler, F.

2001 Mundialización, globalización y sistema capitalista, http://rcci.net/globalizacion/2001/fg155.htm; última visita, 23. de mayo de 2009 .

Taussig, M.

1980 The Devil and Commodity Fetishism in South America. University of North Carolina, Chapel Hill.

Teves, L.

2002 Trazas de paisajes en Molinos, un pueblo del valle Calchaquí, Salta, Argentina. En The Power of Oral History: Memory, Healing and Development, editado por P. Denis y J. Worthington, pp. 145-155. University of Natal, Pietermaritzburg.

White, D.R. y P. Jorion

1996 Kinship networks and discrete structure theory: applications and implications. Social Networks. Special Issue on Discrete Structure Analysis 18:267-314.

Wolf, E.

1997 Europe and the People Without History. University of California, Berkeley, Los Angeles, London.

Zorn, E.

1998 (Re) Fashioning the self: Dress, economy, and identity among the Sakaka of Northern Potosí, Bolivia. Chungara 30:161-196.

\section{Notas}

1 En este artículo se aplica el término telar tradicional para designar a los instrumentos andinos para tejer. El término telar horizontal o telar tradicional no corresponde de un modo adecuado a los aspectos básicos de estos instrumentos. Un telar es un conjunto de instrumentos y utensilios formando un mecanismo que permite abrir las caladas automáticamente. Esta característica básica no es compartida por los instrumentos para tejer, ya que son construidos sin este mecanismo. Ver también el diccionario de ergología y tecnología publicado por Feest (1999).

2 Para aclarar la diferencia entre los términos globalización y mundialización ver el aporte de Soler (2001): "Entendemos, en primer lugar, por "globalización" un fenómeno esencialmente económico que podría concretarse, en una primera 
aproximación, como el proceso de integración económica internacional que tiene como rasgos característicos la liberalización de los mercados, fundamentalmente, pero no sólo, el financiero y, en consecuencia, la profunda financiarización de la economía. ... Así, lo que se llama mundialización no tiene sentido más que si por ella entendemos la aniquilación de los últimos sectores que todavía escapaban a la dominación del capital".

3 Las explicaciones presentadas a continuación parten de datos primarios recabados durante distintas estadías en esta comunidad, la primera entre 1985 y 1989 y la última en julio y agosto del año 2003. Queda claro que dentro del contexto de un artículo sólo es posible presentar una parte de la información. En consecuencia, en el texto sólo se hará referencia a los datos relacionados directamente con el tema y se indicarán las respectivas obras donde se encuentran más detalles en las notas a pie de página (ver Fischer 1999, 2007). Con las demás obras básicas respecto a los temas "tejidos" y "región Charazani" se procede de modo igual. La región Kallawaya se ubica en dirección nordeste del lago Titicaca y abarca los valles interandinos de la Cordillera Real cerca del Nudo de Apolobamba. La comunidad Upinhuaya se localiza en su parte norteña. Es la única comunidad de aymara hablantes (en lo referente a la estructura y la ortografía del idioma hablado entre los miembros del grupo local ver Hardman et al. 1988) dentro de esta región poblada por quechuahablantes.

Para evitar reiteraciones en las bibliografías y citas de mis artículos, un proceder que llevaría a la formación de un cártel de citados, aprovecho para remitir en este lugar a las obras de Saignes (1985, 1986b) y de Girault (1969), ambos escribieron libros clásicos sobre esta región. Sin embargo, para el presente artículo éstas no proveen las informaciones requeridas para el análisis planificado.

4 "Art-any art-requires a comprehensive framework to understand its subtleties, but to understand textiles - potentially the most subtle of personal arts-requires a comprehension of the social life from which these textiles acquire meaning. Such comprehension must take into account the techniques and materials used in the production of cloths, their religious significance and social use, the variety of historical-and indeed, prehistoric-influences on these clothes, their development in time, the range of variation within the tradition, and finally, the intentions of the artists who conceive, create, and produce the cloth. With this background, it is possible to appreciate the composition and quality of individual pieces of textile art" (Fox 1979:39).

5 Ver los documentos del ABNB, EC 1765[1574] n 131:80, 80 a y 96 .

6 Se define criollo como categoría social con características particulares, y no como categoría biológica (Larson 1999:558, nota a pie de página, ver también la opinión crítica de Wolf 1997:X, XIII).

7 Para mayor detalle respecto a esta temática muy amplia ver Fischer 1999 y 2007.

8 Sobre el problema de la definición de "lo andino" comprendido generalmente como "lo tradicional y genuino prehispánico” ver Izko (1986:60).

9 El término creolisation fue desarrollado en el contexto de “... historically recognized creole cultures, mostly in the plantation areas of the New World, but now we sense that "creole cultures" may be turning into more of a generic term, of wider applicability” (Hannerz 1996:66). También se aplica para definir sociedades rurales.

10 En estudios recientes, los datos que toman referencia a las múltiples funciones de los tejidos y a la calidad de la materia prima, llevan a cuestiones sobre el modo de vida, la existencia y la calidad de las relaciones socioeconómicas que establecen las interacciones entre los actores y, finalmente, a cuestiones sobre los elementos que actualmente participan en la construcción de la identidad local. En este contexto se deben recomendar los estudios realizados por Teves (2002) de la Universidad de La Plata (Buenos Aires, Argentina), quien investiga las actividades textiles mediante el análisis de redes sociales (Social Network Analysis/SNA) en Molinos, región andina en el noroeste de Argentina.

11 (...) y al mismo tiempo a las intervenciones de los EE.UU. para borrar por completo las plantaciones de los arbustos de coca del mapa boliviano.

12 En el año 2003 existía una sola tienda para satisfacer los deseos de los comuneros, la que estaba abierta en las tardes.

13 El artículo de Teves (2002) ya indicado nos puede servir para comprender mejor el valor de estas relaciones locales y vinculadas a las redes sociales, tanto las regionales como las transregionales y, en algunos casos, las mundiales.

14 Las hebras se componen por dos o más hilos.

15 Para conseguir información complementaria sobre la indicación de estatus, de género y de identidad por medio de los colores y diseños ver Fischer 2007.

16 A primera vista, los diseños utilizados por las tejedoras originarias de distintas comunidades parecen iguales. Pero examinándolos más precisamente se notan diferencias en lo referente a la preferencia individual de algunas representaciones y a la preferencia colectiva de determinados colores. La preferencia individual de los diseños lleva a la detección de los lazos de parentesco, ya que la transferencia de los diseños se da en línea materna. La preferencia colectiva para determinados colores lleva a la identificación del lugar de origen de una persona. Para mayor detalle ver Fischer 2007.

17 El espacio a disposición para el presente artículo no permite explicar de manera exhaustiva las afinidades, diferencias y problemas en particular existentes y relativos a estos dos ejemplos. Los impulsos externos que llevaron a la comercialización de los tejidos en la isla de Taquile, por ejemplo, tendrán que ser tratados en otro artículo.

18 En el año 2000, con la meta de iniciar una producción de tejidos para el mercado nacional e internacional de artesanía, se llevó a cabo este proyecto iniciado por un equipo alemán.

19 Colorante obtenido de insectos pequeños (Dactylopius coccus cacti) que da, según el mordiente utilizado, diferentes matices de color rojo y violeta.

20 Se trata de la sustancia tintórea añil (Indigofera suffructurosa) cuya denominación proviene de la lengua chiriguana y designa una planta endémica de la región del Chaco (Cajías y Fernández 1987:58).

21 Por la reducida concentración del oxígeno en las regiones altas, se necesita más tiempo y paciencia para el teñido de azul con este colorante. 